\title{
7 The Future and Impact of Adaptive Places
}

This book has presented an approach to designing adaptive places in 3D virtual worlds using grammars and computational agents. Our approach to adaptive virtual worlds is distinctive, and this reflects on the following two aspects.

Our approach associates computational agents with virtual world occupants, rather than virtual world objects. Generative Design Agents (GDAs) serve as personal design agents to users of virtual worlds that can reason, design and act on their behalf in virtual worlds. Shifting the agency from existing virtual world objects to virtual world occupants frees virtual worlds from being static by providing a reasoning process for the world to adapt to users' needs. GDAs perceive a virtual world as a dynamic and evolving entity in three different perspectives: external, internal, and expected $\left(\mathrm{W}=\mathrm{W}_{\text {ext }} \mathrm{U} \mathrm{W}_{\text {int }} \mathrm{U} \mathrm{W}_{\text {exp }}\right)$. By applying generative design grammars, these computational agents dynamically design virtual worlds in response to their use.

Our approach changes the roles of human designers in designing virtual worlds. Designers develop generative design grammars that describe and generate different design languages with different purposes and stylistic characterizations for different virtual places, rather than pre-defining every detail of all possible designs. The GDAs can automate the actual design tasks by applying generative design grammars within virtual worlds in real time. This approach turns designing virtual worlds into a unique process integrated into the virtual world that is highly adaptive and responsive to the changing needs in the virtual worlds.

In this chapter, we explore several considerations for the development and implementation of adaptive virtual places using design rules and agents, and discuss the future and impact of adaptive virtual worlds.

\subsection{Technical Environments for Adaptive Virtual Places}

Throughout the book, we have illustrated our approach in the context of 3D virtual worlds. However, our approach can be applied more generally in light of the many technical environments for designing virtual places. While we focus on 3D virtual worlds, the approach can generalize to any online environment: a web environment such as a networked game, a mobile device for accessing social media places, a mixed reality environment, and a large public interactive display. For example, if the virtual place is expressed on a web site, the layout, object placement, interaction and navigation can be achieved with a design grammar and agent implemented using:

- HTML to specify the categories of content is equivalent to types of objects in a 3D virtual world such as walls, doors, floors, picture frames, etc.;

- XML and files of different media such as images, videos, sound, and 3D models to specify specific content items is equivalent to the 3D models and media in a virtual world;

(c) BY-NC-ND $@ 2014$ Ning Gu and Mary Lou Maher This work is licensed under the Creative Commons Attribution-NonCommercial-NoDerivs 3.0 License. 
- CSS to specify the style rules for each content element or class of elements is equivalent to the layout rules in the virtual world grammar;

- JavaScript to define the local client interactivity is equivalent to the interaction rules that are specific to one user in the virtual world grammar; and

- $\quad$ PHP to describe the shared interactivity shared with other users is equivalent to the navigation and interaction rules that are common to all users.

So, while our development of the design grammar and agent model for adaptive virtual worlds was inspired by place design in 3D virtual worlds, the models are independent of the technology associated with 3D virtual worlds.

\subsection{Functional Designs vs. Creative Designs}

Generative design grammars provide a computational approach that can be adopted to start formally defining design languages for virtual worlds. In our demonstration of an adaptive virtual galley, the example grammar describes and generates a specific language of virtual gallery designs by capturing specific stylistic characterizations through layout rules, object design rules, navigation rules and interaction rules. These four sets of design rules are developed to define a way virtual gallery designs are composed, according to specific design considerations, in terms of both syntax (visualization: layout and object design), and semantics (navigation and interaction). However, the structure of the grammars, the design operations adopted in the rules and the constraints for directing their applications can be flexibly adjusted to address different design considerations. One example consideration is to prioritize the functional aspect and/or the creative aspect of the generated designs, to suit specific purposes for networked gaming, social networking, online learning, e-commerce, collaborative design and so on.

Generative design grammars both adopt the descriptive and generative nature of shape grammars, and modify some of the original shape grammar properties to suit the purpose of designing 3D virtual worlds. As discussed in Chapter 3, currently the application of a generative design grammar is controllable and predictable to a certain extent so that the designed virtual places will be functional and capable of supporting the intended activities. In other words, currently the purpose of generating virtual world designs that meet the GDAs' design goals has been given higher priority over retaining design ambiguity and emergence during the grammar application, which is essential for enabling novel and unexpected designs. However, for virtual worlds that have less functional demand, generative design grammars can be less restricted to enable more creative and diverse virtual world designs or better balance of both the functional and creative aspects.

Adaptive virtual worlds can be highly functional, as they can be perceived as a kind of architecture customized to every "moment", because the virtual worlds are 
dynamically designed and re-designed as needed during use. To more precisely and appropriately meet the actual needs, the knowledge required for the GDA's reasoning process particularly interpretation and hypothesizing should be carefully defined and integrated. The application of a generative design grammar is always directed to meet the current design goals hypothesized by the GDA that reflect its interpretation regarding the current needs of the virtual world users and the current state of the virtual world. It is especially subtle for the GDA to interpret the current needs of the virtual world users. A series of future research issues may arise from here; for example, how should a GDA interpret? By receiving instructions from different virtual world occupants explicitly, by guessing the occupants' intentions based on their activities or from the agent's own learning experience? How precise can and should the interpretation be? What kind of interpretation should lead to changes of virtual world designs? The list of questions can continue.

Further, the design rules in current generative design grammars and the constraints for directing their applications are pre-defined by human designers. If a design grammar needs to address different design considerations or to capture different design characterizations, currently these changes will need to be made by designers through changing the components in the grammar. In future studies, more dynamic alternatives can be explored. For example, machine learning or case-based reasoning can be integrated into the approach to enable GDAs to learn from their past design experiences, and use these experiences to enrich and revise generative design grammars automatically, in order to better address different functional and/or creative design considerations.

\subsection{Individual, Shared and Collective Design Styles}

A generative design grammar describes and generates virtual world designs through the four sets of design rules, therefore the generated designs share a certain design style in terms of visualization, navigation and interaction that are defined by these rules. The current categorization of the design rules provides a base to further detail and expand these stylistic characterizations for defining other virtual world design styles.

In our approach, the agent model currently focuses on defining the five internal computational processes that are specifically developed for a GDA to reason, design and act in 3D virtual worlds. Designing virtual worlds has so far been explored as individual activities carried out by a single GDA. Currently in a collaborative virtual world with the presence of multiple GDAs, when a design problem arises it is assumed that a dominant GDA will reason, design and act on behalf of all occupants. However, the reasoning mechanism of our agent model enables each GDA to reason about the virtual worlds as well as different elements in the virtual worlds, including other GDAs. This means that the GDA model is able to accommodate agent communication. With 
agent communication, virtual worlds can be designed collaboratively by multiple GDAs. Agents are especially suitable for engineering complex, distributed systems. (Jennings, 2000). By providing GDAs with the capability of collaborative designing, a virtual world becomes a distributed system designed by multiple GDAs. Each GDA contributes to the design of the virtual place supporting their shared activities. During collaborative designing, participating GDAs may each have a different generative design grammar that can produce virtual place designs in a unique design style. This leads to the study of shared design styles for virtual worlds. Various research questions can arise from this future direction. For example, how should individual design styles influence each other? How should different design styles transform and morph to accommodate shared interests and preferences? Should there be a common design style shared by a virtual community, and what is the relation and effect of this shared style for individual styles and identities?

In more recent years the term "collective intelligence" (Lévy, 1997) and its potential application in design i.e. "collective design" (Maher et al, 2010) has appeared widely over a broad spectrum of scientific, sociological and design studies, due to the popularity and adoption of social media and social network amongst a unprecedented large number of participants. Collective design is an emerging application of collective intelligence that utilizes very large networked communities in the design process (that has been traditionally reserved for exclusive domain experts) for solving complex problems in potentially innovative ways. 3D virtual worlds as a social medium have been regarded as a possible open-source design environment that can support these collective design processes with massive participation. In the context of collective design, the issues discussed above regarding shared design style will need to be further scaled up to consider collective design style.

\subsection{Extending Adaptive Virtual Places into the Built Environments}

The place metaphor is an important concept in our approach to designing 3D virtual worlds. With the place metaphor, we relate designing these networked environments to designing places in the physical world, considering particularly their layout, object design, navigation and interaction. Although designing virtual places has been heavily influenced by designing physical places, the characteristics of virtual worlds being dynamic, adaptive, and interactive can be equally influential to current and future place design in the physical world, providing an ideal test bed for exploring new and innovative place design such as liquid architecture (Novak, 1991), hyper architecture (Puglisi, 1999), and information architecture (Schmitt, 1999), minimising the risk of safety and cost before being implemented as built environments. With the advancement and increased adoption of digital and networked technologies, we are also seeing the gradual blending of both physical and virtual places such as those 
examples of galleries and museums as discussed in Chapter 5 that have presence in both the physical and virtual worlds.

As a test bed for exploring new and innovative place design in the physical world, adaptive virtual places are especially relevant to interactive architecture (Fox \& Kemp, 2009). Interactive architecture is concerned with the built environments we inhabit as the interactive interfaces between us and the embedded digital information and computational intelligence. "Sensitivity", "smartness", and "responsiveness" are the main characteristics of interactive architecture that support "smart and sustainable living” (Jeng, 2012). The design agent model of this book will provide a formal structure to explore and test the reasoning mechanism required for the place to sense and respond. The generative design grammar framework will provide a rule-based design approach to create and/or modify the components of the place as needed.

\section{References}

Fox, M. and Kemp, M. (2009), Interactive Architecture, Princeton Architectural Press, New York.

Jeng, T. (2012), Interactive Architecture: Spaces that Sense, Think, and Respond to Change, in N. Gu and X. Wang (eds), Computational Design Methods and Technologies: Applications in CAD, CAM and CAE Education, IGI-Global, Hershey, PA, pp. 257-273.

Jennings, N.R. (2000), On Agent-based Software Engineering, Artificial Intelligence, 117 (2), pp. 277-296.

Lévy P. (1997), Collective Intelligence: Mankind's Emerging World in Cyberspace, Perseus Books, Cambridge, MA.

Maher, M.L., Paulini, M. and Murty, P. (2010), Scaling Up: From Individual Design to Collaborative Design to Collective Design, Proceedings of DCC 10, Stuttgart, Germany, pp. 581-600.

Novak, M. (1991), Liquid Architecture in Cyberspace, in M. Benedikt (ed), Cyberspace: First Steps, MIT Press, Cambridge, MA, pp. 225-254.

Puglisi, L.P. (1999), Hyper Architecture: Spaces in the Electronic Age, Birkhäuser, Basel, Switzerland.

Schmitt, G. (1999), Information Architecture: Basis and Future of CAAD, Birkhäuser, Basel, Switzerland. 